\title{
Corrigendum: LncRNA MSC-AS1 Is a Diagnostic Biomarker and Predicts Poor Prognosis in Patients With Gastric Cancer by Integrated Bioinformatics Analysis
}

Wei Yang ${ }^{1 \dagger}$, Fusheng $\mathrm{Ge}^{1 \dagger}$, Shuaibing Lu ${ }^{1 \dagger}$, Zhiming Shan ${ }^{2}$, Liangqun Peng ${ }^{1}$, Junhui Chai ${ }^{1}$, Hongxing Liu ${ }^{1}$, Baodong $\mathrm{Li}^{1}$, Zhandong Zhang ${ }^{1}$, Jinxi Huang ${ }^{1}$, Yawei Hua ${ }^{1 *}$ and Yonglei Zhang ${ }^{1 *}$

${ }^{1}$ Department of General Surgery, The Affiliated Tumor Hospital of Zhengzhou University, Henan Cancer Hospital, Zhengzhou, China, ${ }^{2}$ Clinical Laboratory, The Children's Hospital of Henan Province, Zhengzhou Children's Hospital, The Affiliated Children Hospital of Zhengzhou University, Zhengzhou, China

Keywords: IncRNA MSC-AS1, prognosis, gastric cancer, bioinformatics, biomarker

\section{OPEN ACCESS}

Approved by:

Frontiers Editorial Office,

Frontiers Media SA, Switzerland

${ }^{*}$ Correspondence:

Yawei Hua

huayawei1965@163.com

Yonglei Zhang

xiaoym128910@163.com

†These authors have contributed equally to this work

Specialty section

This article was submitted to Gastroenterology,

a section of the journal

Frontiers in Medicine

Received: 25 January 2022

Accepted: 27 January 2022

Published: 17 February 2022

Citation:

Yang W, Ge F, Lu S, Shan Z, Peng L.

Chai J, Liu H, Li B, Zhang Z, Huang J,

Hua $Y$ and Zhang $Y$ (2022)

Corrigendum: LnCRNA MSC-AS1 is a

Diagnostic Biomarker and Predicts

Poor Prognosis in Patients With

Gastric Cancer by Integrated

Bioinformatics Analysis.

Front. Med. 9:862010.

doi: 10.3389/fmed.2022.862010

\section{A Corrigendum on}

LncRNA MSC-AS1 Is a Diagnostic Biomarker and Predicts Poor Prognosis in Patients With Gastric Cancer by Integrated Bioinformatics Analysis

by Yang, W., Ge, F., Lu, S., Shan, Z., Peng, L., Chai, J., Liu, H., Li, B., Zhang, Z., Huang, J., Hua, Y., and Zhang, Y. (2021). Front. Med. 8:795427. doi: 10.3389/fmed.2021.795427

Shuaibing Lu was not listed as a co-first author in the published article. The corrected author list appears below.

Wei Yang ${ }^{1 \dagger}$, Fusheng $\mathrm{Ge}^{1 \dagger}$, Shuaibing Lu ${ }^{1 \dagger}$, Zhiming Shan ${ }^{2}$, Liangqun Peng ${ }^{1}$, Junhui Chai ${ }^{1}$, Hongxing Liu ${ }^{1}$, Baodong $\mathrm{Li}^{1}$, Zhandong Zhang ${ }^{1}$, Jinxi Huang ${ }^{1}$, Yawei Hua ${ }^{1 *}$ and Yonglei Zhang ${ }^{1 *}$

In the published article, there was an error in affiliation 1. Instead of "Department of General Surgery, Henan Cancer Hospital, Affiliated Tumor Hospital of Zhengzhou University, Zhengzhou, China," it should be "Department of General Surgery, The Affiliated Tumor Hospital of Zhengzhou University, Henan Cancer Hospital, Zhengzhou, China.”

The authors apologize for these errors and state that they do not change the scientific conclusions of the article in any way. The original article has been updated.

Publisher's Note: All claims expressed in this article are solely those of the authors and do not necessarily represent those of their affiliated organizations, or those of the publisher, the editors and the reviewers. Any product that may be evaluated in this article, or claim that may be made by its manufacturer, is not guaranteed or endorsed by the publisher.

Copyright $\odot 2022$ Yang, Ge, Lu, Shan, Peng, Chai, Liu, Li, Zhang, Huang, Hua and Zhang. This is an open-access article distributed under the terms of the Creative Commons Attribution License (CC BY). The use, distribution or reproduction in other forums is permitted, provided the original author(s) and the copyright owner(s) are credited and that the original publication in this journal is cited, in accordance with accepted academic practice. No use, distribution or reproduction is permitted which does not comply with these terms. 